VIEWPOINT

\title{
Diagnosing antimicrobial resistance
}

\section{Carey-Ann D. Burnham, Jennifer Leeds, Patrice Nordmann, Justin O'Grady and Jean Patel}

Abstract | Antimicrobial resistance constitutes a global burden and is one of the major threats to public health. Although the emergence of resistant microorganisms is a natural phenomenon, the overuse or inappropriate use of antimicrobials has had a great effect on resistance evolution. Rapid diagnostic tests that identify drug-resistant bacteria, determine antimicrobial susceptibility and distinguish viral from bacterial infections can guide effective treatment strategies. Moreover, rapid diagnostic tests could facilitate epidemiological surveillance, as emerging resistant infectious agents and transmission can be monitored. In this Viewpoint article, several experts in the field discuss the drawbacks of current diagnostic methods that are used to identify antimicrobial resistance, novel diagnostic strategies and how such rapid tests can inform drug development and the surveillance of resistance evolution.

[0]

The evolution of drug resistance mechanisms in pathogenic microorganisms poses a global health crisis, and millions of people are at risk if the problem is not addressed in the laboratory and translated to the clinic. What role do diagnostics have in managing the challenge of antimicrobial resistance?

Carey-Ann D. Burnham. Antibiotic resistance has emerged as a global health crisis and if we cannot reverse the trend we are on a course towards a post-antibiotic era. There are several ways that diagnostic testing can assist in managing this challenge.

At a very high level, rapid near-patient diagnostic assays to distinguish viral from bacterial infections may prevent treatment with unnecessary antibiotics. If a bacterial infection is present, defining the aetiology and antibiotic susceptibility profile is essential to optimize and narrow antimicrobial therapy as quickly as possible. Whereas traditional methods used in clinical microbiology laboratories would typically require $48 \mathrm{~h}$ (or longer) for definitive results, rapid diagnostic methods are becoming available for routine clinical use; some of these methods can provide results within hours. Although rapid tests for infection can have a positive effect at the individual patient level, diagnostics have a broader role in managing antimicrobial resistance. Rapid susceptibility testing is essential to support clinical trials for new anti-infective agents, and aggregate contemporary data on local and regional antimicrobial resistance trends can facilitate antimicrobial stewardship, epidemiological surveillance and epidemiological efforts.

Jennifer Leeds. From my perspective as a leader of an antibacterial drug discovery group in the pharmaceutical industry, diagnostics enable the appropriate use of new drugs when they are introduced into clinical practice and throughout their commercial lifespan. It is important for medical staff and treating physicians to understand which drugs will benefit an individual patient, and it is important for the antimicrobial stewardship committees to understand how to address overall patient needs at their institutions with respect to antimicrobial resistance. Without a diagnostic device that indicates whether a patient is likely to benefit from a specific drug, the drug could be misused or not used at all, even if it was available and able to save the lives of patients. Appropriate use is a cornerstone in the management of antimicrobial resistance, as it limits the exposure of patients and environments to antibacterials under only those circumstances in which the drugs are likely to improve the health status of an individual. The more inappropriately antibacterials are used, the more we risk the erosion of the utility of a drug due to resistance. With the immense amount of time and resources that it takes to discover new safe and effective antibiotics, diagnostics help the medical community to preserve the utility of these precious drugs.

Patrice Nordmann. Diagnostic techniques may contribute to the identification of rapidly emerging resistance traits. First, such a rapid identification can contribute to better antibiotic stewardship. Rapid adaptation of the antibiotic therapy to the resistance phenotype of the infecting organism may save the lives of patients, as it has been shown that the optimization of the antibiotic therapy during the first $6-12 \mathrm{~h}$ of infection is crucial for the treatment of life-threatening infections. This is particularly true for infections caused by Gram-negative bacteria, such as those species that are currently the main focus of antibiotic resistance research. For example, species in the Enterobacteriaceae family that may produce extended-spectrum $\beta$-lactamases that confer resistance to extended-spectrum cephalosporins, carbapenemases that confer resistance to carbapenems (imipenem, meropenem or ertapenem), or that are resistant to polymyxins. Second, the rapid identification of resistance traits may contribute to the identification of patients who are infected with resistant pathogens but do not show symptoms, particularly in hospital settings. In turn, the immediate isolation of an infected individual in healthcare facilities could prevent the development of outbreaks that are associated with multidrug-resistant bacteria and save costs.

Justin O'Grady. Rapid and comprehensive diagnostics are key for managing antimicrobial resistance; they enable the improved management of patients who are infected, improved antimicrobial stewardship and the development of new antimicrobials. The first clinical question is whether the 
patient needs antimicrobial therapy, and, second, if a microbial infection is present, what antimicrobial is appropriate for successful treatment. Any delay in appropriate antimicrobial therapy can lead to increased mortality in the case of certain infections; for example, ventilator-associated pneumonia and sepsis ${ }^{1}$. The rapid identification of pathogens and antimicrobial resistance or susceptibility will change the way clinicians manage infection, reducing the duration of empirical therapy and facilitating the early initiation of targeted narrow-spectrum treatment with proven antibiotic activity against the causative agent of infection. The ultimate goal for rapid diagnostics should be the identification of the pathogen and antimicrobial susceptibility directly from clinical samples at point of care within approximately $30 \mathrm{~min}$. However, currently, the fastest diagnostic testing methods still take hours, and results are unlikely

\section{The contributors*}

Carey-Ann D. Burnham is an associate professor of pathology and immunology, molecular microbiology, and pediatrics at Washington University School of Medicine, St. Louis, Missouri, USA. She is also the Medical Director of Clinical Microbiology for Barnes Jewish Hospital, St. Louis, Missouri, USA. Her research programme focuses on the development of diagnostic assays for infectious diseases and the transmission and epidemiology of antimicrobial resistance in bacteria.

Jennifer Leeds is an Executive Director and head of Antibacterial Discovery in the Infectious Diseases Area at the Novartis Institutes for Biomedical Research, Emeryville, California, USA, where she is responsible for the strategy and execution of the new antibacterial portfolio, from target discovery through to clinical validation. She is an expert in bacterial genetics and physiology, antibacterial mechanisms of action and resistance, drug discovery, clinical microbiology and clinical development. She co-invented and co-led the international discovery project team for the novel antibacterial LFF571, which demonstrated safety and efficacy in humans for the treatment of infection with Clostridium difficile. Recently, her group disclosed the preclinical discovery programme for LYS228, a novel antibacterial in phase 1 that is being developed to treat infections caused by carbapenem-resistant members of the Enterobacteriaceae. She represents Novartis in drafting and reviewing recommendations for vehicles to incentivize antimicrobial research and development, and is a member of the International Federation of Pharmaceutical Manufacturers Associations (IFPMA) Antimicrobial Resistance (AMR) Research Working Group. She has chaired several recent meetings and symposia on antimicrobial research and development and antimicrobial resistance, and is a member of the Finance Committee of the American Society for Microbiology. She received her Bachelor's degree in microbiology from Cornell University, Ithaca, New York, USA, and her Ph.D. in medical microbiology and immunology from the University of Wisconsin-Madison, USA. Prior to joining the pharmaceutical industry in 2001, she was a postdoctoral fellow in microbiology and molecular genetics at Harvard Medical School, Boston, Massachusetts, USA, where she trained in the laboratory of Jon Beckwith.

Patrice Nordmann is Chair of Microbiology in the Department of Medicine, and Head of the Emerging Antibiotic Resistance Unit and of the Foreign Research Unit INSERM (Paris, France) at the University of Fribourg, Switzerland. He is co-author of more than 680 peer-reviewed publications. His research focuses on the emerging antibiotic resistance traits in Gram-negative bacteria, from fundamental genetics to biochemistry and clinical applications. His latest research topics include carbapenemases and acquired resistance to polymyxins in Gram-negative bacteria.

Justin O'Grady carried out his Ph.D. (2003-2006) and first postdoctoral position (2006-2008) at the National University of Ireland, Galway, followed by a 2 year period in industry at Beckman Coulter (2008-2010), Galway, Ireland, all of which were in the area of infection diagnostics. He subsequently took up a postdoctoral position at University College London, UK, where he focused on tuberculosis diagnostics. In 2013, he was appointed Assistant Professor in Medical Microbiology at Norwich Medical School, University of East Anglia, UK, and was promoted to Associate Professor in 2016. His research focuses on the rapid diagnosis of infectious disease and antimicrobial resistance using molecular methods, with a recent focus on nanopore metagenomic sequencing. Justin O'Grady's homepage. https://www.uea.ac.uk/medicine/people/profile/justin-ogrady

Jean Patel is a clinical microbiologist who has specific expertise in the laboratory detection of antimicrobial resistance. She has experience leading the CDC Antimicrobial Resistance Laboratory and prior to working for the CDC she worked as the Assistant Director of the Clinical Microbiology Laboratory at the Hospital of the University of Pennsylvania, Philadelphia, USA. She has served as chair and vice chair of the Clinical and Laboratory Standards Institute Subcommittee for Antimicrobial Susceptibility Testing and works with the WHO to develop technical guidance for detecting resistance and strengthening the global surveillance of antimicrobial resistance.

*Listed in alphabetical order.

to be available before the administration of empirical treatment, particularly when dealing with life-threatening infections. Providing a comprehensive microbiological diagnosis before the second dose of empirical treatment may be sufficient to improve patient outcomes and stewardship until more rapid diagnostic technologies are available. Moreover, rapid and comprehensive diagnostics will enable the development and use of narrow-spectrum antimicrobials by providing pathogen and resistance information in hours rather than days, and enabling early targeted therapy.

Jean Patel. Antimicrobial use is a primary driver of resistance. The CDC estimates that the use of $50 \%$ of antibiotics (antimicrobials used to treat bacterial infections) administered in hospitals is inappropriate or unnecessary. New diagnostics could fundamentally change when and how antibiotics are used. A diagnostic test that could accurately differentiate a bacterial infection from a viral infection or non-infectious condition would eliminate most of the unnecessary use of antibiotics. A test that could rapidly determine whether a bacterial infection was likely to respond to a specific antibiotic (that is, if it is susceptible) would mean that doctors could pick an appropriate drug faster, thereby improving patient outcomes, decreasing the risk of adverse drug events and reducing the potential for pathogen spread. Another crucial role for diagnostics is identifying patients who are colonized, but may not be infected, with resistant bacteria. These test results can identify hidden reservoirs for transmission, especially in healthcare settings.

What are the drawbacks of the methods currently used to identify antimicrobial resistance?

\section{C.-A.D.B. Most of the commercially} available rapid methods for identifying antimicrobial resistance are genotypic methods - that is, methods that rely on the detection of resistance genes or gene products. There are several limitations to these approaches. The first is that genotypic methods can be effective at predicting antimicrobial resistance, but they do not inform antimicrobial susceptibility. In addition, with these approaches 'you only get what you look for' (typically a small panel of resistance determinants is evaluated). Furthermore, not all resistance phenotypes are conferred by a straightforward genotype, 


\section{PERSPECTIVES}

and genotypic methods would not detect emerging resistance patterns for which the genotype has not been defined.

Phenotypic methods have the advantage of predicting both resistance and susceptibly in a relatively unbiased way. However, microbial growth-based methods have the major disadvantages of requiring a relatively large number of viable organisms for analysis and of the methods being slow. In addition, these growth-based methods are best suited for central laboratory testing and not near-patient testing. Furthermore, although phenotypic methods used in clinical laboratories today produce reproducible results ${ }^{2,3}$, these in vitro assays cannot approximate the complex ecology of infection and do not account for other factors that can influence the outcome of infection, such as host response, biofilm formation, interactions with other microorganisms and bioavailability in tissue.

A logistical challenge is that when new anti-infective agents are cleared for use, there may be a great lag (sometimes years) before methods to measure in vitro susceptibility are developed and/or before these laboratory reagents have regulatory clearance for routine diagnostic use. A lack of methods for routine susceptibility testing may limit the clinical use of these new agents.

Last, there are some important gaps in diagnostic methods; for example, we have very limited tools for the diagnosis of fungal and mycobacterial infections, and even fewer for the rapid detection of antimicrobial resistance in these pathogens. Another example is drug-resistant Neisseria gonorrhoeae infections, which are an urgent public health threat. Although rapid molecular assays exist that provide sensitive and specific detection of this pathogen in clinical specimens, the detection of antimicrobial resistance genes is not a component of these assays. A potential downside to these rapid molecular methods is an absence of culture isolates for susceptibly testing, which could be important both for the care of an individual patient but also for gathering population-level surveillance data.

J.L. For many infection types, the turnaround time for diagnosis and the implementation of diagnostic information is rather slow, so changes from empirical to targeted treatment, or inappropriate to appropriate therapy, may not occur on an effective timescale. This could limit the benefit of niche or narrow-spectrum agents, which are a growing emphasis within the antibacterial discovery and development arena, in clinical practice. In addition, it is not straightforward to add new drugs to existing testing device platforms, so the availability of all approved test formats for a particular drug typically does not coincide with the introduction of a new drug onto the market. This potentially limits the use of the new drug, even when otherwise appropriate. Finally, the current FDA guidelines for antimicrobial susceptibility testing devices licensed for use in the United States do not allow the use of these devices to provide susceptibility data for organisms that are not part of the approved indication for a new drug ${ }^{4}$, so the use of a drug to treat pathogens that are otherwise sensitive to an antibiotic is hampered by the lack of available susceptibility testing for those pathogens that might be treatable with a particular drug on the basis of preclinical and non-clinical data.

P.N. The techniques that are currently used for the identification of antibiotic resistance (broth dilution and disk diffusion techniques) are sometimes time-consuming, expensive and not broad enough. Many diagnostic techniques used for antibiotic susceptibility testing still require culturing of the infective agent, which can take at least $6-18 \mathrm{~h}$. Such culture-dependent methods can thus delay the choice of an appropriate antimicrobial therapy; that is, the choice of the antibiotic to which the bacteria is susceptible. Many molecular techniques are already available for the detection of several resistance traits. However, their main drawbacks are that they are still expensive, they only detect known genes, they cannot be used directly for many clinical samples that contain too few bacterial isolates (for example, for blood samples), and the presence of a resistance gene does not necessarily correlate with the resistance phenotype. In fact, gene expression level and combined mechanisms of resistance may make those resistance traits difficult to predict through the use of molecular techniques alone. Indeed, several and different combined biochemical mechanisms may provide the same resistance phenotype.

J.O'G. In light of rapidly increasing antimicrobial resistance, current culture-based methods are no longer fit for purpose for the diagnosis of infection, particularly acute infection, owing to the slow turnaround time of results. Until relatively recently, empirical broad-spectrum treatment worked for the vast majority of infections, and culture results were only used clinically when treatment failed. Increasing antimicrobial resistance has changed this situation, highlighting the need for rapid diagnostics. Increasing antimicrobial resistance is also forcing changes in recommended first-line treatments, replacing drugs to which pathogens have developed resistance with drugs that pathogens are susceptible to but are often less effective or cause unwanted adverse effects. An example includes the recent change from using trimethoprim to using nitrofurantoin for the treatment of uncomplicated urinary tract infections in the United Kingdom; nitrofurantoin must be avoided in patients with reduced kidney function owing to poor activity (reduced renal elimination of nitrofurantoin, leading to a decreased concentration in the urinary tract) and toxic adverse effects (increased concentration of the drug in the blood). Another example is the switch from using ciprofloxacin to cephalosporins for the treatment of gonorrhoea, as cephalosporins are less effective for the treatment of pharyngeal infection. Trimethoprim or ciprofloxacin could still be used in the approximately $60 \%$ of infections caused by susceptible pathogens ${ }^{5,6}$, if these could be identified in a timely fashion using rapid diagnostics. PCR tests, particularly more recent sample-to-answer multiplex PCR technologies (for example, Biofire Filmarray and Curetis Unyvero panels), are becoming more commonly used in clinical microbiology. However, the resistance markers on these panels are not sufficiently comprehensive to provide clinically actionable results in most cases and their future use is questionable, as they are used in parallel to culture-based methods but cannot replace them.

J.P. Current testing methods for selecting an effective antibiotic can be too slow or provide limited information. Specifically, traditional phenotypic susceptibility testing results are usually available $36-72 \mathrm{~h}$ after the sample is collected from the patient. This is too slow to help with initial treatment decisions and, because of the delay, results are often not used to de-escalate or update the treatment plan when such action is warranted. Molecular tests, such as PCR or hybridization tests for resistance mechanisms, are much faster, with turnaround times of 1-4h. These tests can be very useful for treatment decisions for infections caused by Gram-positive bacteria, such as Staphylococcus aureus, because the number of resistance mechanisms in this species is limited. By contrast, resistant Gram-negative bacteria, such as species in 
the Enterobacteriaceae, Acinetobacter spp. and Pseudomonas spp., have many different resistance determinants (resistance genes and mutations), and often the phenotypic resistance results from a combination of determinants that encode resistance mechanisms acting together at different sites in the bacterial cell. As resistance is so complex, tests that detect only a subset of resistance mechanisms provide limited information for picking a therapeutic agent.

\section{What novel diagnostic strategies and approaches can be implemented to identify antimicrobial drug resistance? What parameters need to be considered when developing phenotypic and genotypic tests for the rapid and accurate detection (or confirmation) of antimicrobial resistance and when selecting useful therapeutic agents?}

C.-A.D.B. There are several novel strategies that can be implemented. It is well established that outpatient visits for respiratory infections are the setting in which the most unnecessary antimicrobials are prescribed. Near-patient testing to rule out bacterial infection or confirm viral aetiology may be a simple approach to reducing the unnecessary use of antimicrobials.

In the setting of serious bacterial infection, rapid phenotypic methods are needed. The ideal method would provide definitive results directly from the clinical specimen within $5-8 \mathrm{~h}$ of specimen collection; with these performance characteristics, the second dose of antibiotics administered could be directed therapy.

Although phenotypic methods to detect both resistance and susceptibility to antibiotics are desired, conventional growth-based systems are slow, and attempts to increase the doubling time of microorganisms have resulted in very limited success. Thus, there is a need to focus on the development of new methods to detect this growth with improved sensitivity, an example of which could be measuring metabolic end products as a surrogate for microbial growth. In addition, methods that measure the host response to therapy are desired. For many infections, the optimal duration of antibiotic treatment is not known, but measuring the host response could be an effective tool to establish an appropriate end point to antimicrobial therapy.

Thus, factors that should be considered when developing new assays include sample requirements (that is, can testing be carried out directly on a clinical specimen or on a pure culture of organism), analysis time, the breadth of the panel of microorganisms and antimicrobial agents that can be detected, and the flexibility of the system to expand testing to additional microorganisms and antimicrobial agents as required.

J.L. There are some new rapid image-based technologies that could provide the turnaround of information more quickly in a clinical setting. Current culture-based methods that require isolated colonies to grow before susceptibility data can be acquired can take up to $48 \mathrm{~h}$ or more, depending on the time lag from patient sampling to the start of the clinical microbiology laboratory processes, the testing time and the time to turn data around and have it reviewed by a physician. New methods that can use direct-from-sample testing and that can report drug susceptibility without the need for standard culture techniques may shorten that time window by at least one day, perhaps more. For example, on the basis of the information disclosed on their website, the Accelerate PhenoTest BC kit can identify species from blood culture in less than $90 \mathrm{~min}$ and antibiotic susceptibility in less than $7 \mathrm{~h}^{7}$. There are also some hand-held nucleic acid-based rapid diagnostic devices being developed that may be useful in field settings, especially when the practicality of culture-based diagnostics is not feasible but the likelihood of certain pathogens is high ${ }^{8}$. What parameters need to be considered when developing phenotypic and genotypic tests for the rapid and accurate detection (or confirmation) of antimicrobial resistance and when selecting useful therapeutic agents? To me, as a drug discoverer, the most important aspect of new diagnostic tools is that they provide clarity about when it is appropriate to use a particular agent. However, knowing that an organism contains genes that could confer resistance to a particular drug still does not necessarily inform what that organism is susceptible to. Thus, a treating physician really benefits when they are given a list of agents that will work against a particular pathogen, not just what will not work. To achieve this, a device will need to robustly report susceptibility data, not just the presence of a resistance determinant, to a panel of available drugs. In addition, the presence of genetic material does not always correlate with the expression levels of those genes, nor does it necessarily take into account other intrinsic or acquired mechanisms that contribute to the loss of susceptibility; therefore, a phenotypic test method will often need to be paired with a genotypic method to capture that complexity.
P.N. The novel strategies for detecting emerging resistance traits may be based on molecular biology techniques, immunology, biochemistry and rapid culture techniques. Novel molecular techniques will involve novel machines based mostly on a PCR technique. However, the costs associated with purchasing and maintaining such devices are currently still too high for them to be available to many institutions and hospitals. Following the development and approval, such novel technologies can be used as a point-of-care technology in hospital settings, such as intensive care units, as well as for general healthcare providers in medical practices. The spread of techniques such as the whole-genome sequencing may provide some help for the rapid identification of specific resistance traits. However, whole-genome sequencing takes at least several hours to obtain results. Currently, this technique is widely used to compare susceptible strains that could be a potential source of outbreaks.

Immunological techniques that are based on antibody-antigen reactions are also being developed, as they provide simple, cost effective and convenient solutions for the identification of several antibiotic resistance traits. Moreover, rapid culture methods, such as those for identifying resistance to polymyxins in members of the Enterobacteriaceae (rapid polymyxin NP test), as well as biochemical approaches, such as those used to identify broad-spectrum enzymes (such as extended-spectrum $\beta$-lactamases or carbapenemases, using the rapid ESBL NP test and rapid Carba NP test, respectively), already enable the rapid identification of resistance markers, although the underlying molecular mechanisms of resistance remain unknown. These techniques provide results within $2 \mathrm{~h}$.

Overall, the parameters of any novel diagnostic technique to be studied are sensibility, specificity, and positive and negative predictive values. In addition, a particular concern is that rapid tests should take into account that there is not necessarily a correlation between the resistance phenotype and genotype.

J.O'G. Recently, agnostic metagenomic sequencing has been tested for the rapid and comprehensive diagnosis of pathogens and antimicrobial resistance, with a first trial currently ongoing for hospital-acquired pneumonia. Rapid nanopore metagenomic sequencing can identify a pathogen (bacteria, viruses, fungi and parasites) and its associated resistance 


\section{PERSPECTIVES}

profile within hours (for example, $4 \mathrm{~h}$ for the diagnosis of urinary tract infections ${ }^{9}$ ) in a single test. This is a very powerful technique that is likely to have a big effect on clinical microbiology in the coming years. However, the detection of antimicrobial resistance (rather than susceptibility) is not ideal, as it informs clinicians on what not to prescribe rather than providing clear treatment options. Also, genotypic resistance may not always correlate with phenotypic resistance (for example, resistance related to gene expression levels), and many resistance mechanisms are multifactorial and not yet fully understood. Rapid susceptibility testing directly from clinical samples is challenging, but should be the ultimate goal for diagnostic technologies attempting to replace culture-based methods.

MALDI-TOF and rapid microscopybased susceptibility testing methods are available (for example, Accelerate PhenoTest $\mathrm{BC})$, but these systems currently rely on cultured clinical samples and/or isolates, which are typically only available $24 \mathrm{~h}$ postincubation. Although combining quantitative PCR (qPCR)-based detection (or, in the future, rapid metagenomics) of pathogen nucleic acid and shortened culture of isolates in media containing antibiotics has been shown to be an effective method for testing antimicrobial susceptibility, the requirement for isolated bacteria increases the turnaround time for results. It remains to be seen whether such technologies can be optimized to work directly on clinical samples, particularly those that contain high bacterial loads, such as samples from patients with urinary tract infections.

\section{J.P. Genotypic testing is fast and} reproducible, and whole-genome sequencing is becoming more affordable. Sequencing data can more accurately detect antibiotic resistance because databases that correlate molecular findings to phenotypic resistance, such as ResFinder, are more robust. Sequencing has the additional benefits of providing molecular typing data to help with outbreak detection and helping infection control experts to better understand transmission dynamics. Advances in metagenomic techniques hold promise that we will soon be able to obtain crucial antimicrobial resistance data from slow-growing pathogens, fastidious pathogens or pathogens that are now commonly detected by culture-independent diagnostics, which, although rapid, provide less data-rich results. For these and other reasons, sequencing approaches, including metagenomics, will initially be most important for pathogens such as Mycobacterium tuberculosis, N. gonorrhoeae and Salmonella spp.

Sequencing will be a powerful tool for the rapid identification of antimicrobial resistance, but phenotypic antimicrobial susceptibility testing is likely to remain the 'gold standard' testing strategy for selecting a therapeutic agent. This is because genotypic testing can only tell us about resistance mechanisms and determinants that have already been discovered, which means that new resistance could be missed and spread undetected. Although phenotypic susceptibility methods have slow turnaround times, technological advances are shortening these delays. Recent phenotypic innovations include single-cell light microscopy, mass spectrometry and measuring genomic transcription after antibiotic exposure. These methodological strategies are all faster than waiting for bacteria to grow overnight in the presence of antibiotics. These methods can become even faster if coupled with advances in technologies that capture and test low numbers of infectious agent from clinical specimens. This would eliminate or shorten the incubation time required to have enough bacteria for testing using current methods. The FDA recently approved a phenotypic susceptibility method that can produce results within about $6.5 \mathrm{~h}$ of a positive blood culture $^{10}$. This is a great advancement and hopefully even faster phenotypic methods will be available in the future.

\section{What opportunities are there for academics, clinicians and scientists in industry to work together to innovate and implement diagnostics that improve patient care and public health?}

C.-A.D.B. Translational research - an ongoing process of iteration - is essential to drive the innovation that is needed to improve patient outcomes and public health. For the success of this translation, academics and clinicians will need to work with industry partners to develop and evaluate new products and move them forward towards market, and diagnostic methods need to be developed in conjunction with new antimicrobial agents. Equally important is the need for ongoing surveillance to identify emerging mechanisms of resistance as early as possible and the characterization of resistance mechanisms; this knowledge is essential for the ongoing development of diagnostic methods and antimicrobial agents for emerging resistance mechanisms.
Aside from antibiotics, novel therapeutic approaches are necessary. Academics and industry must work together to improve our understanding of the microbial disruption that occurs when antimicrobial therapy is administered and what can be done to combat this. Furthermore, an improved understanding of how our resident microbiota contributes to health and disease, and what microorganisms are most important to resistant colonization or infection with pathogens, is an important area of investigation.

J.L. It is an exciting time in antibacterial drug discovery and diagnostic innovation. I attended the American Society for Microbiology (ASM) Microbe meeting 2017 and there was a session called "Bad Bugs, New Drugs — but No Tests!”. The room was packed as a panel consisting of a drug developer, a hospital-based clinical microbiologist, a diagnostic device manufacturer, and an infection control specialist and Jean Patel (an epidemiology expert from the $\mathrm{CDC}$ ), helped the microbiology community to understand the latest developments in the diagnostic device arena. In my opinion, every academic and industry scientist who is innovating diagnostic approaches should attend events such as this one so that they can become aware of the needs and limitations of all aspects of the field. This also gives them a few points of contact with whom to interact and begin to learn more about the practical nature of diagnostics and where innovation can truly have an effect. Working across drug development and device development as a coordinated and collaborative effort is crucial, both from a regulatory and from a practical standpoint. Open lines of communication among clinical laboratory staff, healthcare providers, drug developers and device innovators is crucial to appreciate the goals and the needs of each partner, so that innovators can deliver products that meet and exceed the expectations of the individual stakeholders, and the decision makers who back them, by providing necessary resources. Spending time 'walking in each other's shoes' can teach you a lot about what excites each party and what barriers may lie in the way of a great idea being adopted into clinical practice. Finally, we need to really support training and careers in clinical microbiology. Without trained staff who can implement the use of new devices and provide accurate information back to physicians and other healthcare personnel, the true societal value of new devices is at risk. 
P.N. Academics who are working in association with hospitals have a privileged situation to detect emerging resistance phenomena, as they have access to samples and patients who are infected. On the basis of their observations, they may be in a position to develop specific novel diagnostic tests that correspond to a clinical need, such as the detection of new drug-resistant pathogens. However, the development of resistance tests mostly relies on industry, as the microbiological diagnostic industry has extensive know-how and the money to invest rapidly in the field. As extensively done in the past, clinicians and clinical microbiologists will have a key role in the evaluation and implementation of any novel diagnostic tests that are being developed by industry, and they will have the main role in choosing and retaining the most suitable technique to identify a resistance trait as a source of infection or at the carrier state, based on the clinical value of each test and their cost.

J.O'G. Innovation, translation and implementation skills are all required for new diagnostic tests to reach the bedside; hence, diagnostics innovators in different areas naturally collaborate closely. Recently, governments have provided more funding for research and development into antimicrobial resistance and this has opened many additional opportunities for academic, industrial and clinical innovators to work together. The quality and scope of diagnostic clinical trials have improved with investment, with recent studies considering more than test specificity and sensitivity, and turnaround time, addressing antimicrobial stewardship, cost-benefit analysis and scientist and clinician behaviour in relation to new diagnostics (for example, the INHALE hospital-acquired pneumonia diagnostic trial). These multidisciplinary approaches are crucial if better diagnostics are to be developed and implemented in clinical microbiology. An example of a potential area for future multidisciplinary collaboration is combining the detection of human biomarkers of infection, such as blood transcriptional biomarkers ${ }^{11}$, with the detection of the pathogen and the antimicrobial resistance profile in a single test. For example, using host and pathogen metatranscriptomics for the diagnosis of sepsis could determine whether an infection is present and, if an infection is present, could guide appropriate antimicrobial therapy.

'Big pharma' are pulling out of antimicrobial discovery and development, as they are faced with poor discovery prospects (very few new classes of antibiotic have been discovered since the 1980s) and diminishing returns on investment (the development pipeline is extremely expensive and antibiotics are relatively cheap drugs). Hence, there is a dearth of new antimicrobials in the pipeline. Governments must look beyond big pharma to, for example, small and medium-sized enterprises (SMEs) and universities, who can team up to advance the discovery and development of novel antibiotics and companion diagnostic tests. Clearly, the appropriate mechanisms and government funding need to be in place for this approach to be successful.

\section{J.P. The CDC has several networks for} evaluating and implementing novel diagnostics that will improve antibiotic use and the containment of antibiotic-resistant infections. Specifically, the CDC has a network of academic centres, the Prevention Epicenters, which develops and evaluates innovative ways, including novel diagnostics, to improve antibiotic stewardship and prevent healthcare-associated infections. In addition, the CDC has established the Antibiotic Resistance Laboratory Network (ARLN), which leverages public health laboratory capacity to fill the gaps in testing that is usually carried out in healthcare settings. The ARLN consists of seven regional laboratories for advanced antimicrobial resistance testing capacity, one national tuberculosis whole-genome sequencing laboratory, and additional testing in 57 state and large city laboratories. In the ARLN, the CDC will use innovative tools evaluated by other programmes to improve our ability to generate antimicrobial resistance data for containment and prevention. The CDC is also working to make sure that new diagnostics can detect all types of antibiotic resistance by making ARLN samples of resistant bacteria gathered nationwide available in the $\mathrm{CDC}$ and FDA Antibiotic Resistant Isolate Bank.

\section{Looking beyond the development of diagnostics, what else is needed in the battle against antimicrobial resistance? For example, how will diagnostics inform drug development and the surveillance of resistance evolution in the laboratory? What other tools are needed in this pursuit?}

\section{C.-A.D.B. Even better than rapidly}

diagnosing and treating an infection would be preventing it from occurring in the first place. Vaccines are one component of this, as are infection prevention efforts in both hospital and community settings.
Preventative medicine, in addition to education on health and the appropriate use of anti-infective agents, is fundamental in the battle against the post-antibiotic era. Coordinated, global surveillance and antibiotic-sparing efforts are needed.

Laboratories are challenged to rapidly respond with diagnostic testing methods for emerging resistance mechanisms and pathogens. We should view diagnostics, therapy and antibiotic resistance as a single continuous process. Results of laboratory testing must be integrated into diagnostic pathways; collaboration and integration of laboratory medicine professionals in patient care continuum is essential. Academics and industry must work together to build robust, curated repositories of pathogen sequence data and corresponding phenotypic and clinical data. These data are necessary to realize the potential of sequence-based diagnostics, computational pipelines, and the development of both new diagnostics and new anti-infective agents.

J.L. We need to continue to monitor trends in the epidemiology of resistant pathogens through global surveillance, as well as point prevalence studies. The availability of diagnostics is crucial to help generate meaningful data sets. The data around the epidemiology and spread of resistance are what help drug discoverers and developers to understand current, and predict future, medical need. It takes a long time to discover and bring a new antibacterial drug to market; thus, if we only react once a threat is urgent, we are constantly 'fighting the last war.' The availability of robust diagnostics can keep us ahead of the threat posed by antimicrobial resistance and help us to place resources where they will have the biggest effect with respect to managing resistance. With such accurate and implementable diagnostics, and the necessary information technology (IT) systems to support these efforts, we as drug discoverers would have access to up-to-date information, on a global scale, of where the biggest threats and unmet medical needs lie.

P.N. Beyond the development of diagnostic techniques, the development of novel drugs is of the utmost importance to control the development of antimicrobial resistance. This development will most likely be driven by industry, through the development of novel chemical agents with antibacterial activity. The extensive reports of emerging resistance traits will pave the way for choosing the antibiotic to be developed. Moreover, antimicrobial resistance in 
Gram-negative bacteria will continue to increase, as they emerge in the community setting (in which their spread is difficult or impossible to control) and resistance traits are being transmitted in the hospital settings. It is of vital importance to develop simple computer-based strategies for the identification of patients who are possibly infected with an antimicrobialresistant pathogen at the hospital level to detect possible carriers of antibiotic resistance. For example, in many hospitals worldwide, it is still impossible to know whether the patient has been transferred from a hospital in which the patient could have been infected with a multidrug-resistant bacterium. In addition, the extensive use of precise and rapid diagnostics will facilitate epidemiological surveillance of resistance worldwide in 'real time'. Many important resistance traits are still only being identified after the resistance trait has spread worldwide, as exemplified by the global spread of the extendedspectrum $\beta$-lactamase CTX-M-15, and the carbapenemases OXA-48 and more recently New Delhi metallo- $\beta$-lactamase.

J.O'G. Rapid diagnostics will enable the targeted recruitment of patient samples that contain specific pathogens with specific resistance profiles in clinical trials of antimicrobials and inhibitor combinations. This is currently very important; for example, in the development of $\beta$-lactamase inhibitor combinations. Currently, it is commonplace for more than $75 \%$ of the recruited patients in a trial to be infected with a pathogen that is sensitive to the antibiotic alone, which means that less than $25 \%$ of the samples can be used to test the inhibitor. Moreover, if we can replace culture-based methods with rapid diagnostics, clinicians can prescribe targeted narrow-spectrum agents. This will reinvigorate the antimicrobial development pipeline by creating a more diverse market. Novel narrow-spectrum agents are easier to discover than broad-spectrum agents (as their activity is for a specific pathogen or pathogen group rather than across a broad range of pathogens) and are hence potentially cheaper to develop, but they can only be developed and used if better diagnostics are in place.

The implementation of rapid and comprehensive diagnostics, such as metagenomic sequencing, will change public health globally. Public health agencies will be able to monitor pathogens in real time, making it feasible to track outbreaks as they occur, monitor resistance evolution and spread, identify transmission events, and improve infection control and prevention. The depth and richness of the data will far outweigh current diagnostics data. However, there are many barriers to replacing culture-based methods. Both the personnel and infrastructure need to be in place to handle big data, manage rapid results so that they reach clinicians in a timely fashion and analyse and interpret the sequence data. Governments, regulatory authorities, clinicians and scientists all need to be willing to accept, and to drive, this change.

J.P. A key aspect of our efforts to combat antibiotic resistance is a robust IT infrastructure; this affects all aspects of antimicrobial resistance. In healthcare facilities, we need to ensure that diagnostic tests are rapidly and clearly reported to healthcare providers at the bedside. Too often, laboratory information systems and electronic medical records have limited capability to communicate complex test results. Public health also needs advances in IT. The CDC is addressing this need by building surveillance program to capture both antibiotic use and resistance data from electronic medical records. Healthcare facilities, as well as local, state and regional public health authorities, can use these data to implement containment and prevention measures. The CDC is also working to create interactive tools so that the data are easily accessible by the public, including academic and industry partners who need such data for strategic decision making.

Carey-Ann D. Burnham is at the Department of Pathology \& Immunology, Washington University School of Medicine, 660 South Euclid Avenue, Campus Box 8118, St. Louis, Missouri 63110, USA. cburnham@wustl.edu

Jennifer Leeds is at Novartis Institutes for Biomedical Research, Inc., Infectious Diseases Area, 5300 Chiron Way, Emeryville, California 94608, USA. jennifer.leeds@novartis.com

Patrice Nordmann is at the Medical and Molecular Microbiology Department of Medicine, INSERM European Laboratory (French National Institute for Health and Medical Research, Paris), Natural Reference Center for Emerging Antibiotic Resistance, University of Fribourg, Chemin du Musée 18, 1700 Fribourg, Switzerland. patrice.nordmann@unifr.ch
Justin O'Grady is at Norwich Medical School, Faculty of Medicine and Health Sciences, University of East Anglia, Norwich NR4 7TJ, UK. Justin.OGrady@uea.ac.uk

Jean Patel is at the Antibiotic Resistance Coordination and Strategy Unit, National Center for Emerging Zoonotic and Infectious Diseases, Centers for Disease Control and Prevention, 1600 Clifton Road, Atlanta, Georgia 30329-4027, USA. vzp4@cdc.gov doi: $10.1038 /$ nrmicro.2017.103 Published online 12 October 2017

1. Kuti, E. L., Patel, A. A. \& Coleman, C. I. Impact of inappropriate antibiotic therapy on mortality in patients with ventilator-associated pneumonia and blood stream infection: A meta-analysis. J. Crit. Care 23, 91-100 (2008)

2. Doern, G. V. \& Brecher, S. M. The clinical predictive value (or lack thereof) of the results of in vitro antimicrobial susceptibility tests. J. Clin. Microbiol. 49 S11-S14 (2011).

3. Clinical and Laboratory Standards Institute. Development of in vitro Susceptibility Testing Criteria and Quality Control Parameters. 4th edn (CLSI, 2008)

4. Kircher, S. M. et al. The Susceptibility Testing Manufacturers Association presents an opinion for the delay of current susceptibility tests. Clin. Infect. Dis. 63, 1531-1532 (2016)

5. Kahlmeter, G., Åhman, J. \& Matuschek, E. Antimicrobial resistance of Escherichia coli causing uncomplicated urinary tract infections: A European update for 2014 and comparison with 2000 and 2008. Infect. Dis. Ther. 4, 417-423 (2015).

6. Public Health England. Surveillance of antimicrobial resistance in Neisseria gonorrhoeae: key findings from the Gonococcal Resistance to Antimicrobials Surveillance Programme (GRASP). GOV.UK https://www.gov.uk/ government/uploads/system/uploads/attachment data/ file/567602/GRASP Report 2016.pdf (2016).

7. Accelerate Diagnostics. Accelerate Pheno system. Accelerate Diagnostics http://acceleratediagnostics.com/ products/accelerate-pheno-system/\#features (2017).

8. Nather, D. Inside the Pentagon's biotech strike force. STAT News https://www.statnews.com/2015/11/24/ darpa-biotech-infectious-disease/ (2015)

9. Schmidt, K. et al. Identification of bacterial pathogens and antimicrobial resistance directly from clinical urines by nanopore-based metagenomic sequencing. J. Antimicrob. Chemother. 72, 104-114 (2017).

10. US Food and Drug Administration. FDA allows marketing of test to identify organisms that cause bloodstream infections and provide antibiotic sensitivity results. FDA https://www.fda.gov/NewsEvents/Newsroom/ PressAnnouncements/ucm543150.htm (2017).

11. McHugh, L. et al. A molecular host response assay to discriminate between sepsis and infection-negative systemic inflammation in critically ill patients: discovery and validation in independent cohorts. PLoS Med. 12, e1001916 (2015).

Competing interests statement

The authors declare competing interests: see Web version for details.

\section{Publisher's note}

Springer Nature remains neutral with regard to jurisdictional claims in published maps and institutional affiliations.

\section{FURTHER INFORMATION}

ResFinder: https://cge.cbs.dtu.dk/services/ResFinder/ INHALE hospital-acquired pneumonia diagnostic trial: http://www.ucl.ac.uk/inhale-project/overview Prevention Epicenters:

https://www.cdc.gov/hai/epicenters/index.html Antibiotic Resistance Laboratory Network (ARLN): https://www.cdc.gov/drugresistance/solutions-initiative/ ar-lab-networks.html

CDC and FDA Antibiotic Resistant Isolate Bank: https:// www.cdc.gov/drugresistance/resistance-bank/index.html ALL LINKS ARE ACTIVE IN THE ONLINE PDF 